\title{
Impact of Yoga and Meditation on Cellular Aging in Apparently Healthy Individuals: A Prospective, Open-Label Single-Arm Exploratory Study
}

\author{
Madhuri Tolahunase, ${ }^{1}$ Rajesh Sagar, ${ }^{2}$ and Rima Dada ${ }^{1}$ \\ ${ }^{1}$ Lab for Molecular Reproduction and Genetics, Department of Anatomy, All India Institute of Medical Sciences (AIIMS), \\ New Delhi, India \\ ${ }^{2}$ Department of Psychiatry, All India Institute of Medical Sciences (AIIMS), New Delhi, India \\ Correspondence should be addressed to Rima Dada; rima_dada@rediffmail.com
}

Received 22 September 2016; Revised 17 December 2016; Accepted 22 December 2016; Published 16 January 2017

Academic Editor: Delminda Neves

Copyright (c) 2017 Madhuri Tolahunase et al. This is an open access article distributed under the Creative Commons Attribution License, which permits unrestricted use, distribution, and reproduction in any medium, provided the original work is properly cited.

\begin{abstract}
This study was designed to explore the impact of Yoga and Meditation based lifestyle intervention (YMLI) on cellular aging in apparently healthy individuals. During this 12 -week prospective, open-label, single arm exploratory study, 96 apparently healthy individuals were enrolled to receive YMLI. The primary endpoints were assessment of the change in levels of cardinal biomarkers of cellular aging in blood from baseline to week 12, which included DNA damage marker 8-hydroxy-2'-deoxyguanosine (8$\mathrm{OH} 2 \mathrm{dG}$ ), oxidative stress markers reactive oxygen species (ROS), and total antioxidant capacity (TAC), and telomere attrition markers telomere length and telomerase activity. The secondary endpoints were assessment of metabotrophic blood biomarkers associated with cellular aging, which included cortisol, $\beta$-endorphin, IL-6, BDNF, and sirtuin-1. After 12 weeks of YMLI, there were significant improvements in both the cardinal biomarkers of cellular aging and the metabotrophic biomarkers influencing cellular aging compared to baseline values. The mean levels of 8-OH2dG, ROS, cortisol, and IL-6 were significantly lower and mean levels of TAC, telomerase activity, $\beta$-endorphin, BDNF, and sirtuin- 1 were significantly increased (all values $p<0.05$ ) post-YMLI. The mean level of telomere length was increased but the finding was not significant $(p=0.069)$. YMLI significantly reduced the rate of cellular aging in apparently healthy population.
\end{abstract}

\section{Introduction}

In the last decade there has been a significant increase in complex lifestyle diseases like depression, diabetes mellitus (DM), cardiovascular diseases (CVD), cancer, and infertility. These diseases are strongly associated with accelerated cellular aging $[1,2]$ and have become the bane of modern society [3-5]. Within a homogeneous sample of apparently healthy adult population, biomarkers have been defined recently [6] to characterize the complex processes of accelerated aging phenomenon. Although we do not have any gold standard biomarker to monitor healthy aging, based on the current knowledge of putative biomarkers, the cardinal biomarkers of cellular aging and metabotophic biomarkers which can influence them have become the focus of latest translational research to develop interventions to prevent chronic lifestyle diseases.

The cardinal biomarkers of cellular aging include DNA damage, telomere length attrition, and oxidative stress (OS) [7]. DNA damage causes genomic instability which is responsible for cellular dysfunctions in the pathogenesis of lifestyle diseases [8-10]. OS is the most important cause for DNA damage. Although many different oxidative DNA damage (ODD) products have been identified 8-OH2dG (8-hydroxy2 '-deoxyguanosine), a highly mutagenic oxidative DNA adduct has been the subject of intensive study and is a definitive biomarker of DNA damage [11]. Telomere attrition is due to altered telomere metabolism involving decrease in telomerase enzyme activity and OS. It contributes to genomic instability and is associated with aging and lifestyle diseases [12]. 
Oxidative stress, an imbalance between the prooxidants and the antioxidant defense mechanisms, becomes pathological at both extremes of the physiological range needed for normal cellular functions. It is involved in the pathogenesis of complex lifestyle and chronic diseases [13] including depression [14], obesity [15], and infertility [16, 17], the leading public health problems.

Several metabotrophic blood biomarkers influencing cellular aging include biomarkers of stress and inflammatory response, neuroplasticity, and longevity. Sustained stress response due to chronic stress stimuli causes constantly increased cortisol levels [18], which lead to systemic tissue abnormalities like increased adiposity and neurodegeneration. The level of stress responsiveness (cortisol levels) can be a biomarker for predicting susceptibility to lifestyle diseases [19]. Accelerated aging is characterized by a chronic, lowgrade inflammation ("inflammaging"). Inflammaging is a highly significant risk factor for most of chronic lifestyle diseases [20] and is a potential modifiable target [21]. IL-6 is the most prominent cytokine in inflammaging and is both a marker of inflammatory status and a hallmark of chronic morbidity [22]. Impaired neuroplasticity due to accelerated aging can have negative influence across the entire lifespan [23]. BDNF is a major regulator of neuroplasticity [24], which may be increased in specific regions of the brain by various interventions [25]. Health span and longevity are influenced by several factors. Sirtuin-1 (SIRT1), a histone deacetylase (HDAC), is prominent among them and recently has become a target for various interventions [26]. It systemically influences nutrition and energy metabolism and centrally has a role in circadian rhythm, survival against stress [27] and neuronal plasticity [28].

A variety of interventions have been studied $[29,30]$ to determine their influence on preventing lifestyle diseases and promoting health and longevity. They include drugs targeting specific hallmarks of aging, namely, physical exercise [31], nutrition, caloric restriction [32], and antioxidants [33]. However, no single intervention is shown to be an effective preventive and therapeutic strategy for modern complex lifestyle diseases and provide comprehensive benefits for delaying or reversing accelerated aging. Therefore, further research is needed to find optimum interventions for population at risk of lifestyle diseases. Yoga is an emerging integrative health discipline, which can positively modulate mind and body [34] and has been shown to improve clinical profile of patients with various pathologies [35] including depression, obesity, hypertension, asthma, type II diabetes, and cancer. However, recent reviews on Yoga suggest that potential underlying mechanisms need to be further explored [36]. Studies on biomarkers of disease and health in Yoga based interventions are limited and they have only highlighted diabetic and lipid profiles [37, 38], stress and inflammatory markers [39, 40] and neuroimaging correlates [41], in populations with specific medical conditions. Evidence is lacking regarding the efficacy of Yoga lasting short duration of 3 to 12 weeks in improving the biomarkers of cellular aging in apparently healthy people. Thus, the present study was designed to evaluate the impact of Yoga and Meditation based lifestyle intervention (YMLI) on cellular aging and longevity by analyzing cardinal and metabotrophic biomarkers in the peripheral blood of apparently healthy subjects.

\section{Materials and Methods}

2.1. Study Design and Participants. Ninety-six apparently healthy people were enrolled in this 12-week prospective, open-label, single arm exploratory study, from Aug 2015 to May 2016, designed to explore the impact of YMLI on cellular aging. The key inclusion criteria were male or female aged 30-65 years and leading unhealthy modern lifestyle. The key exclusion criteria were inability to perform the yogic exercises due to any physical challenges and those with recent changes in lifestyle during last 3 months. The study was initiated after ethical clearance (ESC/T-370/22-07-2015) and the registration of the trial with Clinical Trial Registry of India (CTRI REF/2014/09/007532).

\subsection{Procedure}

2.2.1. Yoga and Meditation Based Lifestyle Intervention (YMLI). Eligible subjects were enrolled in the study after screening and baseline characteristics were recorded. Participants underwent 12-week pretested YMLI program comprising theory and practice sessions [42, 43]. YMLI is designed to be an integrative health strategy incorporating the classic components of Yoga including Asanas (physical postures), Pranayama (breathing exercises), and Dhayna (Meditation) which are derived from a mix of Hatha Yoga and Raja Yoga. The YMLI for the current study was suitably modified for apparently healthy subjects. YMLI program included sessions 5 days per week for $12 \mathrm{wks}$. For the first two weeks the sessions were held at integrated health clinic (IHC), AIIMS, New Delhi, and taught by registered, specialized Yoga instructors (educational qualifications include Bachelor of Naturopathy and Yoga Sciences and P.G. Diploma in Yoga Therapy). Remaining 10 weeks were home based. Monitoring of compliance of the home based YMLI was through maintenance of a dairy and telephonic contact. The details of the activities in a day during YMLI program are given in Table 1. Each session in YMLI included a set of Asanas (physical postures), Pranayama (breathing exercises), and Dhayna (Meditation) for approximately 90 minutes. This was followed by an interactive lecture (only during the first two weeks of YMLI at IHC) on lifestyle, lifestyle diseases, and importance of their prevention for 30 minutes.

2.2.2. Laboratory Procedures. During this 12 -week study the participants were evaluated for various biomarkers on day 0 and week 12. Fasting venous blood samples $(5 \mathrm{~mL})$ were collected and divided into two parts. One part was allowed to clot and the serum was separated within $30 \mathrm{~min}$ and the other part was transferred to heparinized/EDTA vials and was centrifuged at $2000 \mathrm{~g}$ for 15 minutes at $4^{\circ} \mathrm{C}$. Both serum and plasma were stored at $-80^{\circ} \mathrm{C}$ until analyzed. ROS detection was done by chemiluminescence assay (Berthold detection luminometer, USA). Peripheral blood leukocyte telomere length was measured by qPCR method and telomerase activity was determined by using a telomerase assay kit (Roche, 
TABLE 1: Details of activities in a day of Yoga and Meditation based Lifestyle Intervention (YMLI) program.

\begin{tabular}{|c|c|c|c|c|}
\hline S. No. & \multicolumn{2}{|c|}{ Practice to be done } & & \multirow{2}{*}{$\begin{array}{c}\text { Duration } \\
5 \mathrm{~min}\end{array}$} \\
\hline$(1)$ & Session preparation instructions & & & \\
\hline \multirow{2}{*}{$(2)$} & Prayer & & & $3 \mathrm{~min}$ \\
\hline & Loosening practices (warm-up) & & & $5 \mathrm{~min}$ \\
\hline \multirow{12}{*}{ (3) } & \multirow{12}{*}{ Asanas (Postures) } & \multirow{3}{*}{ Supine } & Shavasana & $2 \mathrm{~min}$ \\
\hline & & & Uttanpadasana & $2 \mathrm{~min}$ \\
\hline & & & Pawanmuktasana & $2 \min$ \\
\hline & & \multirow{3}{*}{ Prone } & Makarasana & $2 \min$ \\
\hline & & & Bhujangasana & $2 \min$ \\
\hline & & & Salabhasana & $2 \min$ \\
\hline & & \multirow{3}{*}{ Sitting } & Vakrasana & $2 \mathrm{~min}$ \\
\hline & & & Ardha-Matsyendrasana & $2 \min$ \\
\hline & & & Vajrasana & $2 \mathrm{~min}$ \\
\hline & & \multirow{3}{*}{ Standing } & Tadasana & $2 \mathrm{~min}$ \\
\hline & & & Vrikshasana & $2 \mathrm{~min}$ \\
\hline & & & Ardhachakrasana & $2 \mathrm{~min}$ \\
\hline$(4)$ & Relaxation & & Shavasana & $5 \mathrm{~min}$ \\
\hline \multirow{5}{*}{ (5) } & \multirow{5}{*}{ Pranayama (Breathing Exercises) } & & Nadishodhana & \multirow{5}{*}{$20 \mathrm{~min}$} \\
\hline & & & Bhramri & \\
\hline & & & Shitkari & \\
\hline & & & Shitali & \\
\hline & & & Brahmamudra & \\
\hline$(6)$ & Aumkar recitation & & & $3 \min$ \\
\hline$(7)$ & Dhyana (Meditation) & & & $20 \mathrm{~min}$ \\
\hline$(8)$ & Shanti mantra & & & $5 \mathrm{~min}$ \\
\hline$(9)$ & $\begin{array}{c}\text { Interactive session (first } 2 \text { weeks only at } \\
\text { Integrated Health Clinic, AIIMS, New } \\
\text { Delhi) }\end{array}$ & & & $30 \mathrm{~min}$ \\
\hline Total & & & & $120 \mathrm{~min}$ \\
\hline
\end{tabular}

Switzerland), as per manufacturer's protocol. 8-OH2dG was estimated in white blood cell DNA (Cayman's EIA kit). ELISA kits were used for levels of TAC (Cayman Chemical, Ann Arbor, USA), cortisol (DRG Diagnostic, Germany), $\beta$-endorphin (Phoenix Pharmaceuticals, Inc.), IL-6 (GenProbe, Diaclone Diagnostic, France), BDNF (Raybiotech, Inc), and sirtuin-1 (Quayee Bio-Technology). Quality-control assays for biomarkers and validation were performed.

2.2.3. Endpoints. The primary endpoint was to assess the change in levels of cardinal biomarkers of cellular aging from baseline to week 12 . The biomarkers included the following: 8-OH2dG, ROS, and TAC (markers of OS and ODD) and telomere attrition markers telomere length and telomerase activity. The secondary endpoints were assessment of metabotrophic blood biomarkers associated with cellular aging, which included cortisol, $\beta$-endorphin, IL-6, BDNF, and sirtuin-1 from baseline to week 12 .

2.3. Statistical Analysis. Data were analyzed using SPSS 20 (IBM Corp, Armonk, NY). Descriptive statistics are reported as means and standard deviations. Changes in outcome variables were analyzed using paired-samples $t$-test. Exploratory analysis included comparisons for within gender subgroups using paired-sample $t$-test. Significance was accepted at $p<$ 0.05 .

\section{Results}

The flow diagram of participation details is provided in Figure 1. Of 96 subjects, 94 subjects were assessed for impact analysis. Two subjects were excluded from analysis due to poor compliance to the program. Baseline sociodemographic characteristics are shown in Table 2.

After 12 weeks of YMLI, there was significant improvement in both cardinal and metabotrophic biomarkers of cellular aging compared to baseline values (Table 3 ). The mean levels of $8-\mathrm{OH} 2 \mathrm{dG}$ and ROS were significantly lower and mean levels of TAC and telomerase activity were significantly increased (all values $p<0.05$ ). The mean level of telomere length was increased but finding was not significant $(p=$ 0.069). The mean levels of cortisol and IL-6 were significantly 


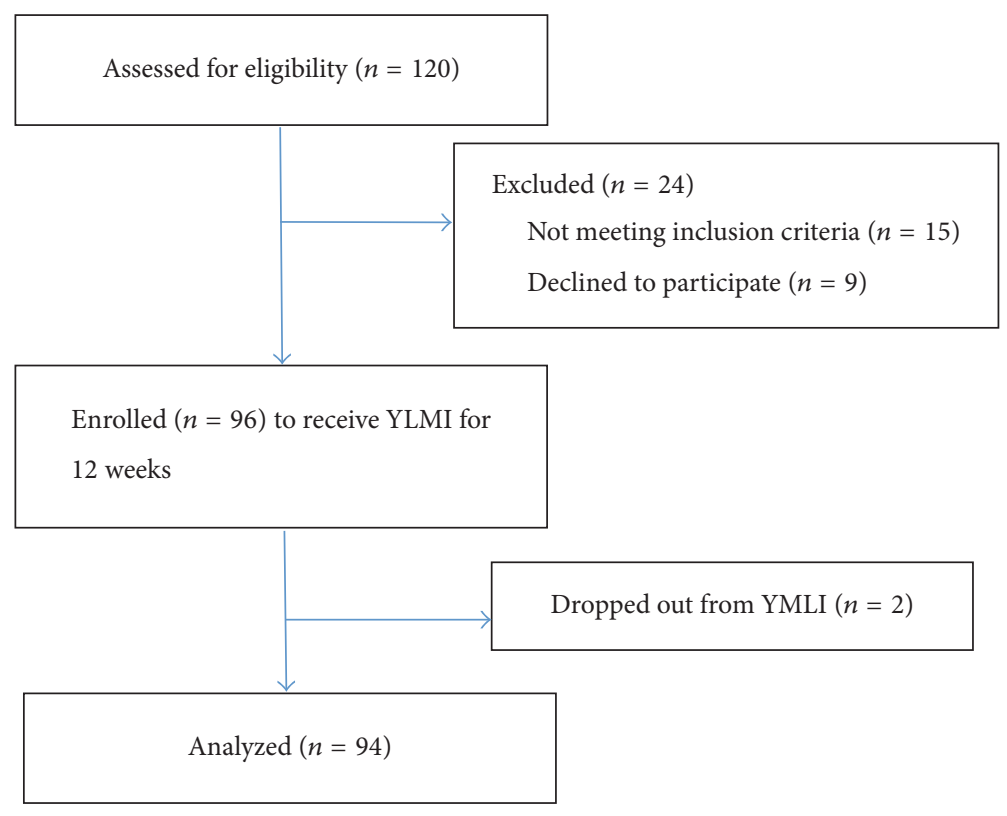

FIGURE 1: Flow diagram of study participation.

TABLE 2: Sociodemographic characteristics of participants.

\begin{tabular}{lc}
\hline Variable & Values \\
\hline Age (years) & $\mathbf{4 0 . 2 6 ( 1 0 . 1 3 )}$ \\
Sex & \\
$\quad$ Female & $\mathbf{5 2}(\mathbf{5 5 . 3 2})$ \\
$\quad$ Male & $\mathbf{4 2 ( 4 4 . 6 8 )}$ \\
Socioeconomic status & \\
$\quad$ Kuppuswamy socioeconomic status scale & \\
$\quad$ Education & $4.82(1.24)$ \\
$\quad$ Occupation & $5.26(2.38)$ \\
$\quad$ Income & $8.60(2.74)$ \\
$\quad$ Total & $\mathbf{1 8 . 6 8 ( 7 . 3 4 )}$ \\
BMI $\left(\mathrm{kg} / \mathrm{m}^{2}\right)$ & $\mathbf{2 6 . 3 0 ( 3 . 4 0 )}$ \\
\hline
\end{tabular}

Data were described as frequency (\%) for sex and mean (SD) for others.

lower and mean levels of $\beta$-endorphin, BDNF, and sirtuin-1 were significantly increased (all values $p<0.05$ ).

Few differences were noted in the gender subgroup analysis. Only male subgroup showed significant decrease in the levels of IL- 6 and a more marked reduction in cortisol levels (males $p=0.001$; females $p=0.036$ ). After 12 weeks of YMLI, we also noted significantly reduced BMI in the study population $(p<0.01)$.

\section{Discussion}

The results of this study highlight the positive impact of YMLI on biomarkers of cellular aging and in promoting cellular longevity through changes in both cardinal and metabotrophic biomarkers. The findings suggest that the impact is mediated through improvement in genomic stability, telomere metabolism, and balance of cellular oxidative stress, well-regulated stress and inflammatory responses, and increase in neuroplasticity and nutrition sensing.

Genomic stability is central to cellular longevity and disease-free youthful healthy life and findings from our study suggest the reduction of genomic instability (decreased levels of $8-\mathrm{OH} 2 \mathrm{dG}$ ) by YMLI. Unhealthy social habits (smoking, excess alcohol intake, etc.), sedentary lifestyle, exposure to environmental pollutants, and intake of processed and nutritionally depleted food have taken a toll on human health with onset of lifestyle diseases at a much younger age [3-5]. These environmental and lifestyle factors are responsible for genomic instability [10]. DNA damage to both mitochondrial and nuclear genome from endogenous as well as exogenous insults results in accumulation of genetic aberrations and genome hypermutability [8-10].

This is mainly due to aberrant DNA damage response (DDR) pathway, which is essential for DNA repair and for monitoring genomic integrity. Deficient DNA repair triggers systemic effects to promote pathological aging [10]. Reduction of DNA damage by YMLI suggests potential of yoga in activating DDR pathway to repair genomic damage and improve genomic stability and changes in metabotrophic factors seen in the study may be associated with these benefits.

Maintaining telomere length through regulation of telomere metabolism contributes to genomic stability and reduction in telomere attrition (increase in telomere length and telomerase activity levels) shown by our study after YMLI suggests the potential for yoga in telomere metabolism and cellular longevity. Telomeres, which serve as a biological clock, are highly conserved hexameric repeats and maintaining their length is vital for cellular longevity. Telomerase 
TABLE 3: Change in outcomes in apparently healthy sedentary subjects participating in a Yoga and Meditation based lifestyle intervention $(n=94)$.

\begin{tabular}{|c|c|c|c|c|c|}
\hline Characteristics & Baseline & $12 \mathrm{wks}$ & $\begin{array}{c}\text { Change from baseline to } \\
12 \mathrm{wks} \\
\text { (diff. } 95 \% \text { C.I.) }\end{array}$ & Effect size* & $p$ value \\
\hline \multicolumn{6}{|c|}{$\begin{array}{l}\text { Primary endpoints: cardinal biomarkers } \\
\text { of cellular aging }\end{array}$} \\
\hline \multicolumn{6}{|l|}{ Oxidative stress } \\
\hline ROS (RLU/min $/ 10^{4}$ neutrophils) & $1215.069 \pm 88$ & $1020.81 \pm 79$ & $194.3(164,224.5)$ & 0.7 & $<0.0001$ \\
\hline TAC (mmol Trolox equiv/L) & $5.94 \pm 1.52$ & $7.4 \pm 2.1$ & $-1.16(-1.9,-0.41)$ & 0.4 & $<0.001$ \\
\hline \multicolumn{6}{|l|}{ DNA damage } \\
\hline $8 \mathrm{OH} 2 \mathrm{dG}(\mathrm{pg} / \mathrm{mL})$ & $1026.23 \pm 630$ & $790.98 \pm 400$ & $235.3(72.73,397.8)$ & 0.22 & $<0.01$ \\
\hline \multicolumn{6}{|l|}{ Telomere attrition } \\
\hline Telomerase activity (IU/cell) & $1.89 \pm 1.42$ & $2.94 \pm 2.2$ & $-1.05(-1.68,-0.41)$ & 0.3 & $<0.001$ \\
\hline Telomere length (IU/cell) & $2.36 \pm 1.6$ & $2.44 \pm 1.4$ & $-0.08(-0.61,0.45)$ & 0.02 & 0.069 \\
\hline \multicolumn{6}{|l|}{$\begin{array}{l}\text { Secondary endpoints: biomarkers } \\
\text { associated with cellular aging }\end{array}$} \\
\hline Cortisol (ng/mL) & $118.83 \pm 50.50$ & $96.32 \pm 38.6$ & $22.51(7.6,37.42)$ & 0.3 & $<0.01$ \\
\hline Interleukin (IL6) (pg/mL) & $3.16 \pm 2.42$ & $1.94 \pm 2.3$ & $1.22(0.47,1.97)$ & 0.3 & $<0.001$ \\
\hline$\beta$-Endorphins (ng/mL) & $6.2 \pm 3.5$ & $8.2 \pm 4.2$ & $-2(-3.22,-0.77)$ & 0.3 & $<0.001$ \\
\hline $\mathrm{BDNF}(\mathrm{ng} / \mathrm{mL})$ & $19.7 \pm 6.75$ & $37.1 \pm 5.6$ & $-17.4(-19.48,-15.32)$ & 0.7 & $<0.0001$ \\
\hline Sirtuin $(\mathrm{ng} / \mathrm{mL})$ & $26.69 \pm 10.42$ & $40.64 \pm 11.6$ & $-13.95(-23.41,-4.49)$ & 0.5 & $<0.01$ \\
\hline$B M I\left(k g / m^{2}\right)$ & $26.30 \pm 3.40$ & $23.64 \pm 3.55$ & $2.66(0.56,3.12)$ & 0.4 & $<0.01$ \\
\hline
\end{tabular}

${ }^{*}$ Effect size was calculated by dividing change by standard deviation at baseline of the specific outcome and interpreted using Cohen's $d$ (small effect: 0.2 to 0.3 , medium effect: 0.5 , and large effect: 0.8 ).

is an important regulator of telomere length and accurate regulation of its activity, and a correct telomere-telomerase interaction is important to precisely safeguard telomere length and prevent telomere attrition [44]. ODD is prominent among the factors which can adversely affect telomere length [45]. Rapid telomere attrition due to ODD is associated with senescence and related disease conditions [46, 47]. Improved telomere metabolism after YMLI seen in the study may contribute to genomic stability. More research is needed to explore the mechanisms of how yoga and meditation intervention can positively modify telomere metabolism.

Our study suggests that improvement in maintenance of balance in cellular oxidative stress (decrease in ROS and increase in TAC) by YMLI. Supraphysiological ROS levels are due to endogenous and exogenous factors like smoking, excess alcohol consumption, exposure to electromagnetic radiation, infection, xenobiotic exposure, and psychological stress [48]. Even the levels of ROS below physiological limits are deleterious to normal cellular function and maintaining OS at physiological levels is important for cellular longevity. Increased OS causes damage to all molecules, including damage to DNA and telomeres. It also affects signal transduction and gene transcription by causing genome wide hypomethylation [49] and thus causes changes in the epigenome. Regulation of cellular oxidative stress within physiological limits after YMLI suggests the potential of this intervention in protecting cells from OS induced DNA damage and telomere attrition and in reversing epigenetic changes, which are accumulated due to unhealthy lifestyle and adverse environmental conditions. Other studies [50] support these findings and have shown reduced OS upregulation of telomerase activity and decreased ODD after YMLI. To combat OS people use antioxidants without monitoring ROS levels resulting in reductive stress [51] unlike in YMLI which regulates ROS levels so that no redox sensitive physiological functions are impaired.

Modern lifestyle and associated psychological stress have complex interactions with lifestyle habits, environmental conditions, and medical interventions to cause accelerated cellular aging, which adversely affect our mental, physical, and reproductive fitness [3-5]. Improved cellular longevity after YMLI suggests the potential role of Yoga in promoting this fitness. While psychological stress is a major manifestation on mind contributing to increased prevalence of neuropsychiatric disorders including depression, abnormal fat accumulation is a major somatic manifestation contributing to increased prevalence of metabolic syndrome and all the diseases that come under the umbrella of metabolic syndrome including, obesity, DM, and CVD [52]. Other peripheral manifestations of unhealthy modern lifestyle include aging of gonads leading to infertility [53] and recurrent pregnancy loss. Previous studies have demonstrated the clinical benefits of Yoga and Meditation in all these medical conditions [35]. Dada et al. have shown that YMLI can reduce testicular aging and result in significant upregulation in telomerase activity and decline in seminal OS and ODD [8]. The ongoing studies in our laboratory on the impact of Yoga and Meditation have provided significant 
evidence for the reversal of cellular aging in subjects prone to accelerated aging due to depression. Microarray (Agilent $8 \times 60 \mathrm{k}$ Microarray kit) and analysis of gene expression preand post-YMLI showed decreased IL6, IL10, and MAP10 and increased IL2 and IL4 $[8,54,55]$. Improved cellular longevity seen in our study after YMLI suggests that changes in both cardinal and metabotrophic biomarkers of cellular aging may be a mechanism for preventing chronic lifestyle diseases. Our study suggests that the changes in metabotrophic factors, which include increase in levels of $\beta$-endorphin, BDNF, and sirtuin- 1 and decrease in levels of cortisol and IL-6, and the cellular processes involving them, may have important roles in reversal of cellular aging and improving cellular longevity after YMLI.

Improvement in stress and inflammatory response in our study after YMLI may be mediated by changes in cortisol, $\beta$-endorphin, IL- 6 , and other factors, with regulation by changes in brain through hypothalamic-pituitary-adrenal (HPA) axis. The response may involve regulation of adaptive pathways including integrated stress response (ISR) [18], which activate the eukaryotic translation initiation factor 2 alpha (eIF $2 \alpha$ ), that promote cellular recovery driving the signaling toward cell survival and longevity. The response may lead to decreased OS and reversal of senescent secretory phenotype of cells, including cells in brain, adipose tissue, endothelium, and gonads. Changes in secretory phenotype include decreased IL-6 [56], increased BDNF, and sirtuin-1 [57]. These regulated factors may lead to balance in OS and cellular longevity and contribute to tissue revival throughout the body from neuroplasticity in CNS to gonads, vessels, and muscles in the periphery. Secretory phenotype from somatic cells provides regulatory feedback to brain [58], which completes the vicious cycle of regulation between mind and body. Neurodegeneration is associated with pathogenesis of several neuropsychiatric conditions and neuroplasticity has a central role in their management and for vitality. Increased BDNF, sirtuin- 1 , and $\beta$-endorphin and decreased cortisol, which decrease cellular aging in brain, decrease neurodegeneration and increase neuroplasticity [23]. Increased cellular longevity and increased neuroplasticity may be a mechanism for alteration of gray matter volume in different regions of the cerebral cortex [59], increased mindfulness [60], and several other complex processes $[55,61]$ involved in reduction of stress and depression after Yoga and Meditation. Regulated mind-body communications may lead to minimization of subclinical inflammation and activation of nutrition and energy sensing pathways promoting longevity, where decrease in IL- 6 and increase in sirtuin-1 play a prominent role, respectively. Previous studies have demonstrated increase in sirtuin-1 levels after interventions with caloric restriction [62]. Our study is the first to document increase in sirtuin-1 levels independent of caloric restriction after practicing Yoga. These improved processes may result in delaying onset and slowing down progression of diseases associated with accelerated cellular aging.

The impact of the intervention in both genders was assessed separately since men and women respond differently to day to day stress [63]. Interestingly, the gender subgroup analysis showed that reduction in cortisol and IL6 levels were more pronounced in male than in female subjects. No significant gender differences were seen in other biomarkers. Phase of the menstrual cycle should be taken into consideration since some biomarker levels are known to vary with different phases of the menstrual cycle $[64,65]$. Our study showed significant decrease in BMI in apparently healthy subjects which came into normal range $(23.64 \pm 3.55)$ from baseline overweight range $(26.30 \pm 3.40)$. While latest research [66] suggests people with mean BMI of 27 , who are overweight by current classification of obesity, are likely to survive longest in western population, similar data is not available for Indian population. Therefore, our findings need to be interpreted cautiously.

Stratification of cases was not done in this study to do subgroup analysis due to small sample size.

Lifestyle is an integrated entity, and an intervention, like YMLI, that has overall positive influence on our health appears most useful versus changing only one aspect at a time, as is seen by action of certain drugs. Yoga is holistic and a mind-body medicine and is more beneficial and advantageous than individual interventions like physical exercise, caloric restriction, and antioxidants. The practice of Yoga and physical exercise are different entities, the former results in energy conservation with economy of energy expenditure for mental and physical benefits, and the later results in energy expenditure more for physical exertions and metabolic needs, as is evident from a study which showed exercise causes erratic changes in biomarkers and results in OS [67] while Meditation brings about uniform biomarker and behavioral changes and improvement in cognition and decrease OS [68]. Therapeutic antioxidants can only decrease ROS rather than regulating it and may paradoxically shorten life span [69] due to imbalance in ROS mediated immune response [70]. YMLI regulates ROS rather than simply lowering them by balanced stress-related processes and appropriate gene expressions [71]. The only limitation of our study is that it is a single arm proof of concept study and did not include controls. It is important to adopt a lifestyle which slows the decline in health by reversing or delaying accelerated aging due to unhealthy lifestyle. The biomarkers of cellular aging can form the basis for determining the risk of chronic lifestyle diseases and the efficacy and usefulness of interventions to decrease disease risk. Hence, findings from this study are supportive of YMLI as a significant clinical utility especially in prevention of and management of complex multifactorial diseases and reducing the rate of functional decline with aging.

\section{Conclusion}

Though we cannot change our biology or chronological age we can definitely reverse/slow down the pace at which we age by adopting YMLI. This is the first study to demonstrate improvement in both cardinal and metabotrophic biomarkers of cellular aging and longevity in apparently healthy population after Yoga and Meditation based lifestyle intervention. So our health and the rate at which we age entirely depends on our choices. Making Yoga and Meditation an integral part of our lifestyle may hold the key to delay aging or aging 
gracefully, prevent onset of multifactorial complex lifestyle diseases, promote mental, physical, and reproductive health, and prolong youthful healthy life.

\section{Competing Interests}

The authors stated that there is no conflict of interests whatsoever regarding the publication of this paper.

\section{Acknowledgments}

The authors are thankful to Amit Tomar and Sudhir Choudary for Yoga instructions and all the subjects enrolled in the study.

\section{References}

[1] A. S. Jackson, X. Sui, J. R. Hébert, T. S. Church, and S. N. Blair, "Role of lifestyle and aging on the longitudinal change in cardiorespiratory fitness," Archives of Internal Medicine, vol. 169, no. 19, pp. 1781-1787, 2009.

[2] V. Boccardi, G. Paolisso, and P. Mecocci, "Nutrition and lifestyle in healthy aging: the telomerase challenge," Aging, vol. 8, no. 1, pp. 12-15, 2016.

[3] J. K. Chakma and S. Gupta, "Lifestyle and non-communicable diseases: a double edged sword for future India," Indian Journal of Community Health, vol. 26, no. 4, pp. 325-332, 2014.

[4] M. J. Pappachan, "Increasing prevalence of lifestyle diseases: high time for action," The Indian Journal of Medical Research, vol. 134, no. 2, pp. 143-145, 2011.

[5] P. Scarborough, P. Bhatnagar, K. K. Wickramasinghe, S. Allender, C. Foster, and M. Rayner, "he economic burden of ill health due to diet, physical inactivity, smoking, alcohol and obesity in the UK: an update to 2006-07 NHS costs," Journal of Public Health, vol. 33, no. 4, pp. 527-535, 2011.

[6] K.-H. Wagner, D. Cameron-Smith, B. Wessner, and B. Franzke, "Biomarkers of aging: from function to molecular biology," Nutrients, vol. 8, no. 6, article 338, 2016.

[7] C. Correia-Melo, G. Hewitt, and J. F. Passos, "Telomeres, oxidative stress and inflammatory factors: partners in cellular senescence?" Longevity \& Healthspan, vol. 3, no. 1, 2014.

[8] R. Dada, S. B. Kumar, M. Tolahunase, M. Mishra, K. Mohanty, and T. Mukesh, "Yoga and meditation as a therapeutic intervention in oxidative stress and oxidative DNA damage to paternal genome," Journal of Yoga \& Physical Therapy, vol. 5, no. 4, 2015.

[9] M. B. Shamsi, S. Venkatesh, D. Pathak, D. Deka, and R. Dada, "Sperm DNA damage \& oxidative stress in recurrent spontaneous abortion (RSA)," Indian Journal of Medical Research, vol. 133, no. 5, pp. 550-551, 2011.

[10] M.-R. Pan, K. Li, S.-Y. Lin, and W.-C. Hung, "Connecting the dots: from DNA damage and repair to aging," International Journal of Molecular Sciences, vol. 17, no. 5, article 685, 2016.

[11] M. Maes, I. Mihaylova, M. Kubera, M. Uytterhoeven, N. Vrydags, and E. Bosmans, "Increased 8-hydroxydeoxyguanosine, a marker of oxidative damage to DNA, in major depression and myalgic encephalomyelitis / chronic fatigue syndrome," Neuroendocrinology Letters, vol. 30, no. 6, pp. 715-722, 2009.

[12] I. Chiodi and C. Mondello, "Telomere and telomerase stability in human diseases and cancer," Frontiers in Bioscience, vol. 21, no. 1, pp. 203-224, 2016.
[13] N. Khansari, Y. Shakiba, and M. Mahmoudi, "Chronic inflammation and oxidative stress as a major cause of age-related diseases and cancer," Recent Patents on Inflammation \& Allergy Drug Discovery, vol. 3, no. 1, pp. 73-80, 2009.

[14] C. N. Black, M. Bot, P. G. Scheffer, P. Cuijpers, and B. W. J. H. Penninx, "Is depression associated with increased oxidative stress? a systematic review and meta-analysis," Psychoneuroendocrinology, vol. 51, pp. 164-175, 2015.

[15] A. Salmon, "Beyond diabetes: does obesity-induced oxidative stress drive the aging process?" Antioxidants, vol. 5, no. 3, article no. $24,2016$.

[16] K. Verma and G. C. Baniya, "A comparative study of depression among infertile and fertile women," International Journal of Research in Medical Sciences, vol. 4, no. 8, pp. 3459-3465, 2016.

[17] A. Katib, "Mechanisms linking obesity to male infertility," Central European Journal of Urology, vol. 68, no. 1, pp. 79-85, 2015.

[18] K. Pakos-Zebrucka, I. Koryga, K. Mnich, M. Ljujic, A. Samali, and A. M. Gorman, "The integrated stress response," EMBO Reports, vol. 17, no. 10, pp. 1374-1395, 2016.

[19] S. D. Hewagalamulage, T. K. Lee, I. J. Clarke, and B. A. Henry, "Stress, cortisol, and obesity: a role for cortisol responsiveness in identifying individuals prone to obesity," Domestic Animal Endocrinology, vol. 56, pp. S112-S120, 2016.

[20] C. Franceschi and J. Campisi, "Chronic inflammation (Inflammaging) and its potential contribution to age-associated diseases," Journals of Gerontology-Series A Biological Sciences and Medical Sciences, vol. 69, pp. S4-S9, 2014.

[21] F. Prattichizzo, V. De Nigris, L. La Sala, A. D. Procopio, F. Olivieri, and A. Ceriello, “Inflammaging' as a druggable target: a senescence-associated secretory phenotype-centered view of type 2 diabetes," Oxidative Medicine and Cellular Longevity, vol. 2016, Article ID 1810327, 10 pages, 2016.

[22] M. Maggio, J. M. Guralnik, D. L. Longo, and L. Ferrucci, "Interleukin-6 in aging and chronic disease: a magnificent pathway," Journals of Gerontology-Series A Biological Sciences and Medical Sciences, vol. 61, no. 6, pp. 575-584, 2006.

[23] J. Shaffer, "Neuroplasticity and clinical practice: building brain power for health," Frontiers in Psychology, vol. 7, article no. 1118, 2016.

[24] H. Park and M.-M. Poo, "Neurotrophin regulation of neural circuit development and function," Nature Reviews Neuroscience, vol. 14, no. 1, pp. 7-23, 2013.

[25] S. F. Sleiman, J. Henry, R. Al-Haddad et al., "Exercise promotes the expression of brain derived neurotrophic factor (BDNF) through the action of the ketone body $\beta$-hydroxybutyrate," eLife, vol. 5, Article ID e15092, 2016.

[26] M. S. Bonkowski and D. A. Sinclair, "Slowing ageing by design: the rise of $\mathrm{NAD}^{+}$and sirtuin-activating compounds," Nature Reviews Molecular Cell Biology, pp. 679-690, 2016.

[27] A. C. Sánchez-Hidalgo, M. F. Muñoz, A. J. Herrera et al., "Chronic stress alters the expression levels of longevity-related genes in the rat hippocampus," Neurochemistry International, vol. 97, pp. 181-192, 2016.

[28] F. Ng, L. Wijaya, and B. L. Tang, "SIRT1 in the brainconnections with aging-associated disorders and lifespan," Frontiers in Cellular Neuroscience, vol. 9, article no. 64, 2015.

[29] B. K. Kennedy, S. L. Berger, A. Brunet et al., "Aging: a common driver of chronic diseases and a target for novel interventions," Cell, vol. 159, no. 4, pp. 709-713, 2014. 
[30] V. D. Longo, A. Antebi, A. Bartke et al., "Interventions to slow aging in humans: are we ready?" Aging Cell, vol. 14, no. 4, pp. 497-510, 2015.

[31] D. E. R. Warburton, C. W. Nicol, and S. S. D. Bredin, "Health benefits of physical activity: the evidence," CMAJ, vol. 174, no. 6, pp. 801-809, 2006.

[32] L. Vitetta and B. Anton, "Lifestyle and nutrition, caloric restriction, mitochondrial health and hormones: scientific interventions for anti-aging," Clinical Interventions in Aging, vol. 2, no. 4, pp. 537-543, 2007.

[33] V. Conti, V. Izzo, G. Corbi et al., "Antioxidant supplementation in the treatment of aging-associated diseases," Frontiers in Pharmacology, vol. 7, article 00024, 2016.

[34] B. Patwardhan, G. Mutalik, and G. Tillu, Integrative Approaches for Health-Biomedical Research, Ayurveda and Yoga, Academic Press, London, UK, 1st edition, 2015.

[35] A. Büssing, A. Michalsen, S. B. S. Khalsa, S. Telles, and K. J. Sherman, "Effects of yoga on mental and physical health: a short summary of reviews," Evidence-Based Complementary and Alternative Medicine, vol. 2012, Article ID 165410, 7 pages, 2012.

[36] T. Field, "Yoga research review," Complementary Therapies in Clinical Practice, vol. 24, pp. 145-161, 2016.

[37] M. E. Papp, P. Lindfors, M. Nygren-Bonnier, L. Gullstrand, and P. E. Wändell, "Effects of high-intensity hatha yoga on cardiovascular fitness, adipocytokines, and apolipoproteins in healthy students: a randomized controlled study," Journal of Alternative and Complementary Medicine, vol. 22, no. 1, pp. 8187, 2016.

[38] P. M. Siu, A. P. Yu, I. F. Benzie, and J. Woo, "Effects of 1-year yoga on cardiovascular risk factors in middle-aged and older adults with metabolic syndrome: a randomized trial," Diabetology and Metabolic Syndrome, vol. 7, no. 1, article no. 40, 2015.

[39] M.-S. Ha, D.-Y. Kim, and Y.-H. Baek, "Effects of Hatha yoga exercise on plasma malondialdehyde concentration and superoxide dismutase activity in female patients with shoulder pain," Journal of Physical Therapy Science, vol. 27, no. 7, pp. 2109-2112, 2015.

[40] B. Hari Krishna, C. Kiran Kumar, and N. M. Reddy, "Association of leukocyte telomere length with oxidative stress in yoga practitioners," Journal of Clinical and Diagnostic Research, vol. 9, no. 3, pp. CC01-CC03, 2015.

[41] R. Santhakumari, I. Reddy, R. Archana, and P. Rajesh, "Role of yoga in alienating the memory decline and frontal lobe metabolite changes in type 2 diabetes," International Journal of Research in Ayurveda \& Pharmacy, vol. 7, no. 1, pp. 78-81, 2016.

[42] R. L. Bijlani, R. P. Vempati, R. K. Yadav et al., "A brief but comprehensive lifestyle education program based on yoga reduces risk factors for cardiovascular disease and diabetes mellitus," Journal of Alternative and Complementary Medicine, vol. 11, no. 2, pp. 267-274, 2005.

[43] R. Vempati, R. Bijlani, and K. K. Deepak, "The efficacy of a comprehensive lifestyle modification programme based on yoga in the management of bronchial asthma: a randomized controlled trial," BMC Pulmonary Medicine, vol. 9, article no. 37, 2009.

[44] H. Oeseburg, R. A. De Boer, W. H. Van Gilst, and P. Van Der Harst, "Telomere biology in healthy aging and disease," Pflugers Archiv European Journal of Physiology, vol. 459, no. 2, pp. 259268, 2010.

[45] S. Kawanishi and S. Oikawa, "Mechanism of telomere shortening by oxidative stress," Annals of the New York Academy of Sciences, vol. 1019, pp. 278-284, 2004.
[46] M. A. Shammas, "Telomeres, lifestyle, cancer, and aging," Current Opinion in Clinical Nutrition and Metabolic Care, vol. 14, no. 1, pp. 28-34, 2011.

[47] J. Thilagavathi, S. Venkatesh, and R. Dada, "Telomere length in reproduction," Andrologia, vol. 45, no. 5, pp. 289-304, 2013.

[48] M. P. Pruchniak, M. Arazna, and U. Demkow, "Biochemistry of oxidative stress," in Advances in Clinical Science, vol. 878 of Advances in Experimental Medicine and Biology, pp. 9-19, Springer, 2016.

[49] W. Wongpaiboonwattana, P. Tosukhowong, T. Dissayabutra, A. Mutirangura, and C. Boonla, "Oxidative stress induces hypomethylation of LINE-1 and hypermethylation of the RUNX3 promoter in a bladder cancer cell line," Asian Pacific Journal of Cancer Prevention, vol. 14, no. 6, pp. 3773-3778, 2013.

[50] S. B. Kumar, R. Yadav, R. K. Yadav, M. Tolahunase, and R. Dada, "Telomerase activity and cellular aging might be positively modified by a yoga-based lifestyle intervention," The Journal of Alternative and Complementary Medicine, vol. 21, no. 6, pp. 370372, 2015.

[51] A. Rahal, A. Kumar, V. Singh et al., "Oxidative stress, prooxidants, and antioxidants: the interplay," BioMed Research International, vol. 2014, Article ID 761264, 19 pages, 2014.

[52] A. V. W. Nunn, J. D. Bell, and G. W. Guy, "Lifestyle-induced metabolic inflexibility and accelerated ageing syndrome: insulin resistance, friend or foe?" Nutrition and Metabolism, vol. 6, article no. 16, 2009.

[53] J. Thilagavathi, M. Kumar, S. S. Mishra, S. Venkatesh, R. Kumar, and R. Dada, "Analysis of sperm telomere length in men with idiopathic infertility," Archives of Gynecology and Obstetrics, vol. 287, no. 4, pp. 803-807, 2013.

[54] M. R. Tolahunase, R. K. Yadav, S. Khan, and R. Dada, "Reversal of aging by yoga and meditation," Journal of International Society of Antioxidants, vol. 1, no. 1, 2015.

[55] T. Dada, M. A. Faiq, K. Mohanty et al., Eds., Effect of Yoga and Meditation Based Intervention on Intraocular Pressure, Quality of Life, Oxidative Stress and Gene Expression Pattern in Primary Open Angle Glaucoma: A Randomized Controlled Trial, ARVO, Seattle, Wash, USA, 2016.

[56] A. H. Miller and C. L. Raison, "The role of inflammation in depression: from evolutionary imperative to modern treatment target," Nature Reviews Immunology, vol. 16, no. 1, pp. 22-34, 2016.

[57] S.-W. Min, P. D. Sohn, S.-H. Cho, R. A. Swanson, and L. Gan, "Sirtuins in neurodegenerative diseases: an update on potential mechanisms," Frontiers in Aging Neuroscience, vol. 5, article no. 53, 2013.

[58] T. Tchkonia, Y. Zhu, J. Van Deursen, J. Campisi, and J. L. Kirkland, "Cellular senescence and the senescent secretory phenotype: therapeutic opportunities," Journal of Clinical Investigation, vol. 123, no. 3, pp. 966-972, 2013.

[59] C. Villemure, M. Čeko, V. A. Cotton, and M. Catherine Bushnell, "Neuroprotective effects of yoga practice: age-, experience, and frequency-dependent plasticity," Frontiers in Human Neuroscience, vol. 9, article 00281, 2015.

[60] P. L. Dobkin and Q. Zhao, "Increased mindfulness-the active component of the mindfulness-based stress reduction program?" Complementary Therapies in Clinical Practice, vol. 17, no. 1, pp. 22-27, 2011.

[61] K. E. Riley and C. L. Park, "How does yoga reduce stress? A systematic review of mechanisms of change and guide to future inquiry," Health Psychology Review, vol. 9, no. 3, pp. 379-396, 2015. 
[62] G. López-Lluch and P. Navas, "Calorie restriction as an intervention in ageing," Journal of Physiology, vol. 594, no. 8, pp. 2043-2060, 2016.

[63] B. D. Darnall, M. Aickin, and H. Zwickey, "Pilot study of inflammatory responses following a negative imaginal focus in persons with chronic pain: analysis by sex/gender," Gender Medicine, vol. 7, no. 3, pp. 247-260, 2010.

[64] P. A. Nepomnaschy, R. M. Altman, R. Watterson, C. Co, D. S. McConnell, and B. G. England, "Is cortisol excretion independent of menstrual cycle day? A longitudinal evaluation of first morning urinary specimens," PLoS ONE, vol. 6, no. 3, Article ID e18242, 2011.

[65] J. Ferrer, J. Mtnez-Guisasola, F. Díaz, F. Alonso, M. Guerrero, and B. Marín, "Plasma levels of $\beta$-endorphin during the menstrual cycle," Gynecological Endocrinology, vol. 11, no. 2, pp. 75-82, 1997.

[66] S. Afzal, A. Tybjærg-Hansen, G. B. Jensen, and B. G. Nordestgaard, "Change in body mass index associated with lowest mortality in Denmark, 1976-2013," JAMA, vol. 315, no. 18, pp. 1989-1996, 2016.

[67] J. L. Harte, G. H. Eifert, and R. Smith, "The effects of running and meditation on beta-endorphin, corticotropin-releasing hormone and cortisol in plasma, and on mood," Biological Psychology, vol. 40, no. 3, pp. 251-265, 1995.

[68] T. W. W. Pace, L. T. Negi, D. D. Adame et al., "Effect of compassion meditation on neuroendocrine, innate immune and behavioral responses to psychosocial stress," Psychoneuroendocrinology, vol. 34, no. 1, pp. 87-98, 2009.

[69] G. Bjelakovic, D. Nikolova, L. L. Gluud, R. G. Simonetti, and C. Gluud, "Mortality in randomized trials of antioxidant supplements for primary and secondary prevention: systematic review and meta-analysis," JAMA, vol. 297, no. 8, pp. 842-857, 2007.

[70] M. Ristow, K. Zarse, A. Oberbach et al., "Antioxidants prevent health-promoting effects of physical exercise in humans," Proceedings of the National Academy of Sciences of the United States of America, vol. 106, no. 21, pp. 8665-8670, 2009.

[71] S. Kumar, R. Yadav, and R. Dada, "Yoga as an effective lifestyle intervention for Bhopal methyl isocyanate gas leakage catastrophe victims," International Journal of Yoga, vol. 8, no. 2, p. 162, 2015. 


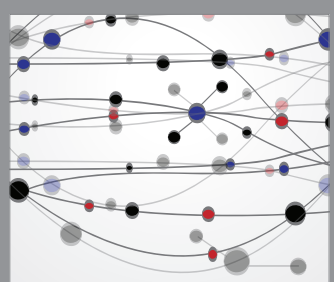

The Scientific World Journal
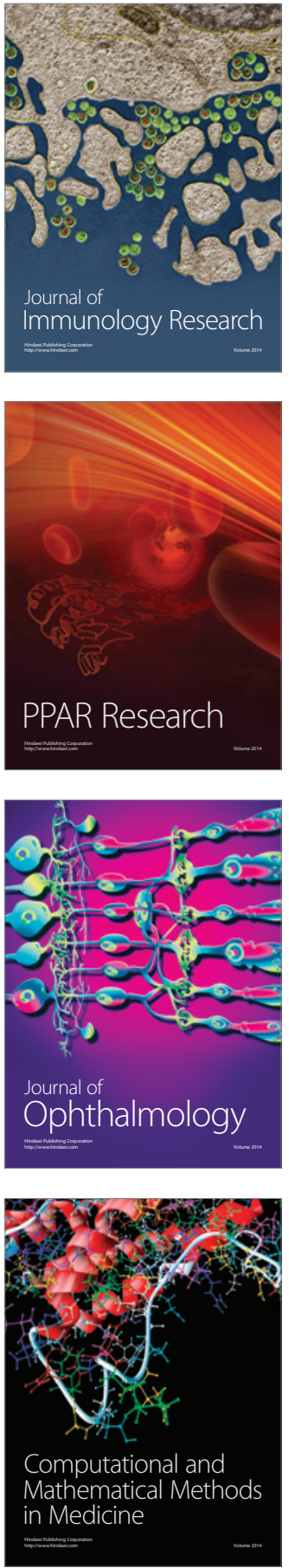

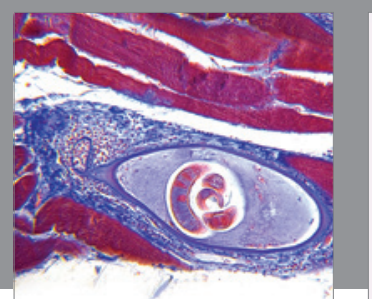

Gastroenterology Research and Practice
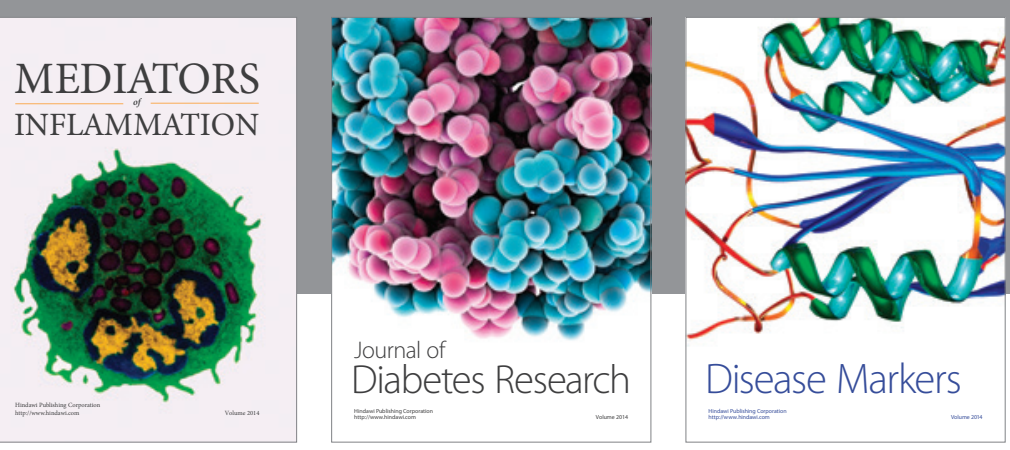

Disease Markers

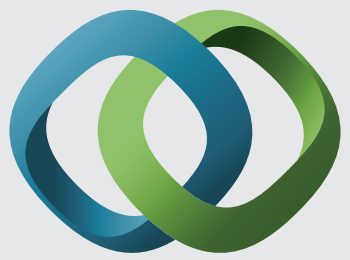

\section{Hindawi}

Submit your manuscripts at

https://www.hindawi.com
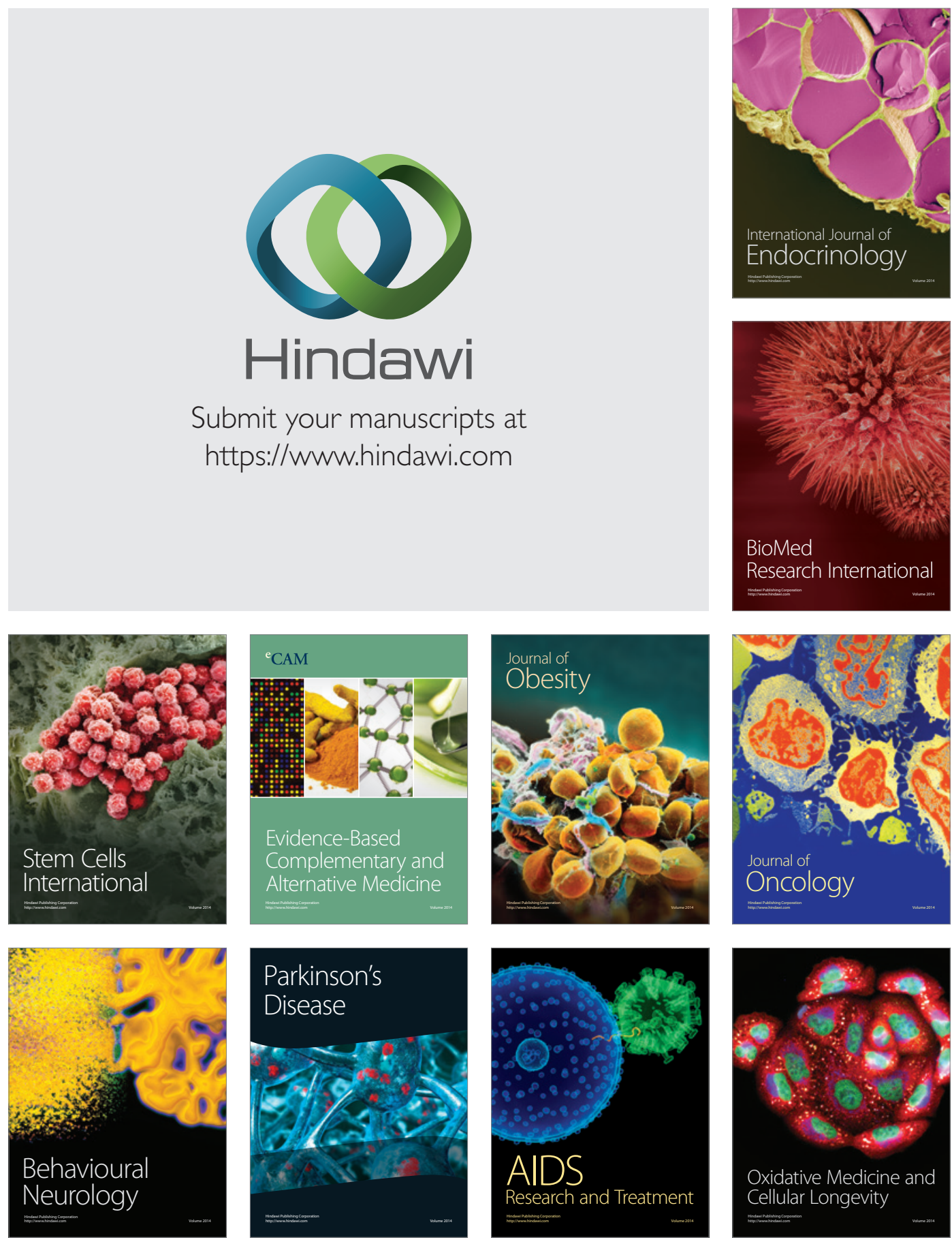